\title{
Changes in lower extremity alignment in standing position using a foot plate
}

\author{
Hye-Mi Lee ${ }^{a}$, Ji-Eun Yang ${ }^{a}$, Ju-Yeon Lee ${ }^{a}$, Hong-Jun Im $^{a}$, Yu-Jin Jeong ${ }^{b}$, Dae-Sung Park ${ }^{a}$ \\ ${ }^{a}$ Department of Physical Therapy, College of Medical Science, Konyang University, Daejeon, Republic of Korea \\ ${ }^{b}$ Department of Medical Science, The Graduate School of Konyang University, Daejeon, Republic of Korea
}

\begin{abstract}
Objective: Eversion of the foot is created with internal rotation of the shank, and inversion of the foot is created with external rotation of the shank. The purpose of the study was to investigate the effect of continuous changes in the angle of the subtalar joint
\end{abstract} on lower extremity alignments.

Design: Cross-sectional study.

Methods: Seventeen healthy young adult subjects recruited. The subjects were asked to stand up in a natural standing position on a footplate with eye open and equal weight on each foot for $10 \mathrm{~s}$ in two different conditions: The right subtalar joint was everted continuously $0^{\circ}-20^{\circ}$ and in separate segments of $0^{\circ}, 5^{\circ}, 10^{\circ}, 15^{\circ}, 20^{\circ}$. The averages of three trials were used. The observation of the changes in the lower extremity was performed with the use of 3-dimensional motion analysis. For data analysis, the SPSS 18.0 software using paired t-test and repeated measures analysis of variance (ANOVA) was applied.

Results: The angle was significantly increased at the horizontal rotation angle of the shank, thigh, and ankle without anterior rotation of the pelvis $(p<0.05)$. The maximum horizontal rotation angle at the thigh on $20^{\circ}$ was $-4.52^{\circ}$ in static, and $-3.10^{\circ}$ in the dynamic conditions compared to $0^{\circ}$.

Conclusions: Increased unilateral foot pronation, thigh, shank, ankle horizontal rotation variance was significantly effective. The observation of the changes in foot abduction with the use of a 3-dimensional motion analysis augmented in predicting the angle values of each segment of the lower extremity. In further studies, a comparison of the right and left subtalar joints need to be investigated.

Key Words: Foot, Kinematic alignment, Subtalar joint

\section{Introduction}

The subtalar joint articulates on three sides that allow for pronation and supination movements of the ankle joint [1]. The ankle joint has a role in maintaining balance in standing and walking, and with pronation and supination movements, the subtalar joint is used in major activities such as walking, changing direction, balance, and also horizontal turning movements of the ankle joint and calf [2]. In addition, other structures of the lower leg influences the pronation and supination of the subtalar joint [3].

Eversion of the foot is created with internal rotation of the shank, and inversion of the foot is created with external rotation of the shank [4,5]. If the supination angle of the foot is increased, the patellofemoral angle and tibiofibular angle decreases. The reason the tibiofibular joint decreases when the foot pronates from a supination position, is because the hip and leg rotate together on an axis [6]. Thus, movement of the foot and alignment of the leg is associated with each other. The degree of internal rotation of the leg and talus bone, when the heel comes in contact with the ground while the subtalar joint is in pronated has not been confirmed in previous studies [7].

In a previous study, muscle activity was significantly re-

Received: 19 August, 2016 Revised: 7 September, 2016 Accepted: 7 September, 2016

Corresponding author: Dae-Sung Park

Department of Physical Therapy, College of Medical Science, Konyang University, 158 Gwanjeodong-ro, Seo-gu, Daejeon 35365, Republic of Korea Tel: 82-42-600-6419 Fax: 82-42-600-6565 E-mail: daeric@naver.com

(c) This is an Open-Access article distributed under the terms of the Creative Commons Attribution Non-Commercial License (http://creativecommons.org/licens es/by-nc/4.0) which permits unrestricted non-commercial use, distribution, and reproduction in any medium, provided the original work is properly cited.

Copyright $@ 2016$ Korean Academy of Physical Therapy Rehabilitation Science 
duced in single limb stance (SLS) on a $15^{\circ}$ wedge rather than SLS without a wedge [8].

In a previous study with use of motion analysis and markers on the thoracic segment, pelvic segment, and heel in SLS with the calcaneous everted at $0^{\circ}, 5^{\circ}$, and $10^{\circ}$, when the heel abduction angle is increased, the movement of pelvic anterior tilt as well as the abduction angle of the hip was increased with leg alignment analysis [9]. In a study that used a footplate in $10^{\circ}, 20^{\circ}$, and $30^{\circ}$ of inversion and eversion, the tibiofibular and tibiocalcaneal angles increased when the foot was in inversion [6]. With use of a wedge to keep the foot fixed in inversion and eversion, a research was done to observe the changes in the body. Although the results were not found to be statistically significant in the amount of pelvic tilt, a decreasing trend was confirmed [9].

In a study with a wedge placed in the front, rear, medial and lateral sides to compare the movements of the leg, the subtalar joint moved 6 times more with a wedge placed in the medial and rear than without use of a wedge, and with a wedge on the medial and rear in the sagittal plane, the ankle and hip joint angles in the frontal plane, the trunk movement moves 2-3 times more than without use of a wedge [10].

In a previous study where a wedge was placed at $10^{\circ}, 15^{\circ}$, and $20^{\circ}$ to assess the alignment of the lower extremity (LE), it was confirmed that as the angle of the wedge increased the shank internally rotates, the femur externally rotates, and the pelvis tilts anteriorly [3].

Excessive eversion of the subtalar joint increases abnormal motion compensation in the knees, pelvis, trunk, and ankle joint [10].

Although there have been studies in differences in wedge angle to assess the LE alignment, continuous changes of pronation of the subtalar joint have yet to be studied.

The purpose of this study was to determine the basis for continuous ankle eversion at $0^{\circ}-20^{\circ}$ as well as study the changes in the femoral, shank, and LE alignment to use as basic data to understand the changes in foot deformity.

\section{Methods}

\section{Subjects}

In this study, the subjects were 17 healthy young adult women that attended Konyang University. The subjects were healthy adults without previous traumatic history on the knees, hips or ankles, and all subjects had normal hip range of motion and no past history of neurological disease. Exclusion criteria included persons with arthritis, back pain, muscle weakness or limited movement. The subjects all signed a consent and voluntarily agreed to participate in this experiment after receiving explanations on the purpose of the study (Table 1).

\section{Assessment tools}

The experimental foot plate that was used was adjustable to control the angle for the entire foot, forefoot, hindfoot, and arch. The footplate moves dynamically and is composed of six plates with two bottom plates that move the entire foot, and two middle plates that can measure the angle of adduction and abduction, and the remaining two plates measure the changes in the hindfoot and midfoot. Each plates is adjustable from $0^{\circ}-20^{\circ}$ (Figure 1).

With the subjects in exercise pants, we placed the markers.

Table 1. Characteristics of subjects

\begin{tabular}{lr}
\hline Characteristic & \multicolumn{1}{c}{ Value } \\
\hline Age $(\mathrm{y})$ & $18.47(0.62)$ \\
Height $(\mathrm{cm})$ & $162.63(5.08)$ \\
Weight $(\mathrm{kg})$ & $58.25(7.22)$ \\
\hline
\end{tabular}

Values are presented as mean (SD).

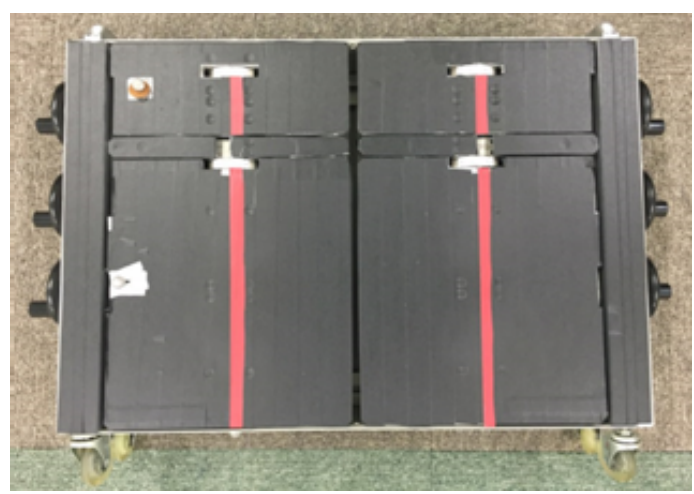

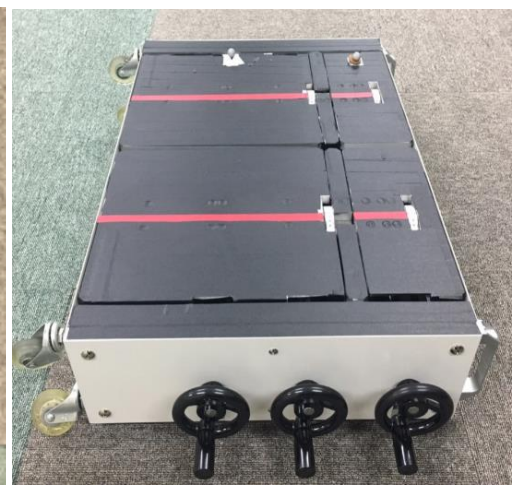

Figure 1. Foot plate of anterior view and lateral view. 
A total of 19 markers were placed bilaterally on the anterior superior iliac spine (ASIS), femoral shaft, medial and lateral condyles, shank shaft, medial, and lateral malleoli, between the second and third toe joints (dorsal), heel, and one placed on the sacrum. The markers on the shaft of the femur and shank were attached to different heights to separate the left from the right.

We collected the movement data with the use of 63 -dimensional infrared motion analysis cameras. The sampling rate was $60 \mathrm{~Hz}$, and we used a Butterworth low pass filter. The cut off frequency was $4 \mathrm{~Hz}$.

\section{Experimental methods}

Subjects were instructed to stand on the footplate after all the markers were in place. Each foot was aligned straight to the footplate with the second toe (Figure 2). The subjects were then instructed to stand with equal weight on each side as possible. The experiment was performed with the subjects eyes open and were instructed to fix their eyes on a target that was $2 \mathrm{~m}$ away from the subject at shoulder height. The arms were in a natural resting position hanging at the sides, and the subjects were asked to move as little as possible. In this position, the right plate which measures the angle of the foot was everted to measure the changes in alignment of the LE.

The right subtalar joint was continuously everted from $0^{\circ}-20^{\circ}$. To compare with previous studies, the averages of three trials of static standing at $5^{\circ}, 10^{\circ}, 15^{\circ}$, and $20^{\circ}$ were used [11].

\section{Data analysis}

IBM SPSS Statistics ver. 20.0 (IBM Co., Armonk, NY, USA) was used for data analysis.

Descriptive analysis was used to compare and analyze the general characteristics

A paired t-test was used to compare the changes in segmental alignment between static and dynamic conditions, and a one-way repeated measured ANOVA was used to compare the LE segments in the dynamic condition. The level of statistical significance was set at $p<0.05$.

\section{Results}

Seventeen healthy young adult women without disability participated in this study in order to evaluate the impact on the femur, shank, foot, and pelvis with continuous eversion.

\section{Dynamic position (change of angle in each segment)}

There was a significant difference in the femoral, shank, and foot angle when the footplate angle was dynamically changed, however no significant differences were found at the pelvis. Post analysis results in the femur in all angles showed significant differences, and there was significant differences at the shank at $5^{\circ}-10^{\circ}, 5^{\circ}-15^{\circ}, 5^{\circ}-20^{\circ}, 10^{\circ}-20^{\circ}$,
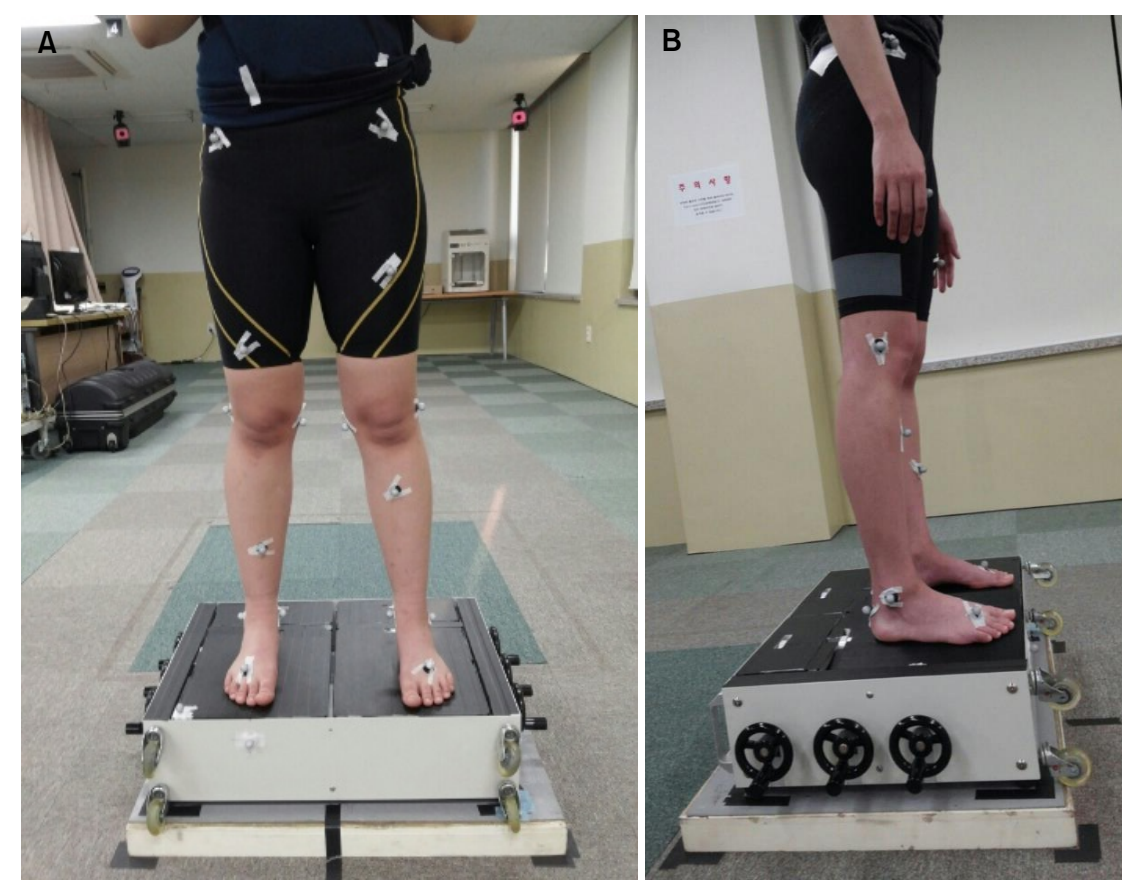

Figure 2. Anterior and lateral view of marker placement during standing on a plate. (A) Anterior view. (B) Lateral view. 
and $15^{\circ}-20^{\circ}$. There was a significant difference in the foot in all angles $(p<0.05$; Table 2$)$.

\section{Static position (change of angle in each segment)}

There was a significant difference in the femur, shank, and foot, however no significant differences were found at the pelvis. Post analysis results showed a significant difference in static changes in the footplate at the femur at $5^{\circ}-10^{\circ}$,

Table 2. Comparison of lower extremity segments in dynamic condition $(\mathrm{N}=17)$

\begin{tabular}{llrrrrl}
\hline \multirow{2}{*}{ Factor } & Segment & $\begin{array}{r}\text { Type III } \\
\text { sum of } \\
\text { squares }\end{array}$ & \multicolumn{1}{c}{$\begin{array}{c}\text { degree } \\
\text { of } \\
\text { freedom }\end{array}$} & F & $p$ \\
\hline Angle & Thigh & 56.121 & 1.872 & 15.627 & $<0.001^{\text {a,b,c,d,e,f }}$ \\
& Shank & 75.207 & 2.187 & 11.357 & $<0.001^{\text {a,c,d,e,f }}$ \\
& Foot & 14.213 & 1.905 & 16.060 & $<0.001^{\text {a,b,c,d,e,f }}$ \\
& Pelvic & 0.252 & 2.332 & 0.754 & 0.496 \\
Error (angle) & Thigh & 57.460 & 29.957 & & \\
& Shank & 105.952 & 34.990 & & \\
& Foot & 14.159 & 30.480 & & \\
& Pelvic & 5.343 & 37.305 & & \\
\hline
\end{tabular}

Dynamic position, one-way repeated measured ANOVA.

${ }^{a}$ Significant difference between $5^{\circ}$ and $10^{\circ}$. ' Significant difference between $5^{\circ}$ and $15^{\circ}$. ${ }^{\mathrm{c}}$ Significant difference between $5^{\circ}$ and $20^{\circ}$. ${ }^{\mathrm{d}}$ Significant difference between $10^{\circ}$ and $15^{\circ}$. ${ }^{\mathrm{e}}$ Significant difference between $10^{\circ}$ and $20^{\circ}$. ${ }^{\mathrm{f}}$ Significant difference between $15^{\circ}$ and $20^{\circ}$. $5^{\circ}-15^{\circ}, 5^{\circ}-20^{\circ}, 10^{\circ}-20^{\circ}$, and $15^{\circ}-20^{\circ}$. There was a significant difference in the shank at $5^{\circ}-15^{\circ}$ and $5^{\circ}-20^{\circ}$. There was a significant difference in the foot at $5^{\circ}-10^{\circ}, 5^{\circ}-20^{\circ}$, and $15^{\circ}-20^{\circ}$ $(p<0.05$; Table 3$)$.

\section{Changes in angle in dynamic and static positions}

When comparing the dynamic and static changes in angle positions of the footplate, not all segments showed a sig-

Table 3. Comparison of lower extremity segments in static condition

$(\mathrm{N}=17)$

\begin{tabular}{llrrrr}
\hline \multirow{2}{*}{ Factor } & Segment & $\begin{array}{c}\text { Type III } \\
\text { sum of } \\
\text { squares }\end{array}$ & $\begin{array}{c}\text { Degree } \\
\text { of } \\
\text { freedom }\end{array}$ & F & $p$ \\
\hline Angle & Thigh & 65.283 & 2.538 & 12.167 & $<0.001^{\mathrm{a}, \mathrm{b}, \mathrm{c}, \mathrm{d}, \mathrm{e}}$ \\
& Shank & 32.898 & 2.270 & 3.165 & $0.048^{\mathrm{d}, \mathrm{f}}$ \\
& Foot & 7.484 & 2.138 & 4.486 & $0.017^{\mathrm{a}, \mathrm{c}, \mathrm{d}}$ \\
& Pelvic & $1,274.010$ & 1.001 & 1.072 & 0.316 \\
Error (angle) & Thigh & 85.848 & 40.605 & & \\
& Shank & 166.335 & 36.320 & & \\
& Foot & 26.692 & 34.214 & & \\
& Pelvic & $19,014.083$ & 16.015 & & \\
& & & & &
\end{tabular}

Static position, one-way repeated measured ANOVA.

${ }^{\mathrm{a}}$ Significant difference between $5^{\circ}$ and $10^{\circ}$. ' Significant difference between $5^{\circ}$ and $15^{\circ} .{ }^{\mathrm{c}}$ Significant difference between $5^{\circ}$ and $20^{\circ}$. ${ }^{\mathrm{d}}$ Significant difference between $10^{\circ}$ and $15^{\circ}$. ${ }^{\mathrm{e}}$ Significant difference between $10^{\circ}$ and $20^{\circ}$. ${ }^{\mathrm{f}}$ Significant difference between $15^{\circ}$ and $20^{\circ}$.

Table 4. Comparison of changes in segmental alignment between static and dynamic condition

$(\mathrm{N}=17)$

\begin{tabular}{lrrrrrr}
\hline Segment & Angle & Static $^{\mathrm{a}}$ & Dynamic $^{\mathrm{a}}$ & Mean difference & $\mathrm{t}$ & \multicolumn{1}{c}{$p$} \\
\hline Thigh & $5^{\circ}$ & $-1.82(2.80)$ & $-0.69(1.14)$ & 1.130 & 1.539 & 0.143 \\
& $10^{\circ}$ & $-2.92(2.78)$ & $-1.55(1.30)$ & -0.090 & -0.107 & 0.916 \\
& $15^{\circ}$ & $-3.53(3.67)$ & $-2.44(1.88)$ & 1.090 & 1.350 & 0.196 \\
& $20^{\circ}$ & $-4.52(3.29)$ & $-3.10(2.10)$ & 1.426 & 1.780 & 0.094 \\
Shank & $5^{\circ}$ & $-0.84(4.12)$ & $0.48(2.29)$ & 1.315 & 1.068 & 0.301 \\
& $10^{\circ}$ & $-1.46(2.98)$ & $-1.17(1.98)$ & 0.294 & 0.275 & 0.787 \\
& $15^{\circ}$ & $-2.42(3.96)$ & $-0.99(2.04)$ & 1.436 & 1.432 & 0.171 \\
Foot & $20^{\circ}$ & $-2.51(3.50)$ & $-0.249(2.33)$ & 0.019 & 0.016 & 0.987 \\
& $5^{\circ}$ & $-0.13(1.08)$ & $-0.20(0.48)$ & -0.071 & -0.253 & 0.804 \\
Pelvic & $10^{\circ}$ & $0.31(1.21)$ & $0.11(0.66)$ & -0.204 & -0.794 & 0.439 \\
& $15^{\circ}$ & $0.36(1.58)$ & $0.59(0.91)$ & 0.228 & 0.703 & 0.492 \\
& $20^{\circ}$ & $0.80(1.70)$ & $0.99(1.29)$ & 0.190 & 0.432 & 0.671 \\
& $5^{\circ}$ & $10.83(30.97)$ & $-0.17(0.46)$ & -11.001 & -1.465 & 0.162 \\
& $10^{\circ}$ & $5.50(29.46)$ & $-0.10(0.62)$ & -5.601 & -0.779 & 0.447 \\
& $15^{\circ}$ & $-0.89(44.24)$ & $-0.11(0.80)$ & 0.772 & 0.072 & 0.944 \\
\hline
\end{tabular}

Values are presented as mean (SD).

${ }^{a}$ Positive values: thigh external rotation, shank external rotation, foot eversion, pelvic posterior tilt; negative values: thigh internal rotation, shank internal rotation, foot inversion, pelvic anterior tilt. 
nificant difference (Table 4).

\section{Discussion}

The purpose of the study was to investigate the effect of continuous changes in the angle of the subtalar joint on LE alignments. Although there are studies that have observed changes in LE alignment according to changes in the subtalar joint angles, studies that have examined the changes in each of the LE segments are insufficient.

Therefore, through this study, the specific correlation in each LE segments with changes in subtalar joint angles have been investigated. In addition, the specific changes in the each segment was compared during static positions.

There were significant differences in the angle of the foot in all of the LE segments from $5^{\circ}-20^{\circ}$ except the pelvis in a static position. When measuring during a continuous dynamic state, there were significant differences from $5^{\circ}-20^{\circ}$.

There was no significant differences in LE segmental changes during the static state and dynamic state with $5^{\circ}$, $10^{\circ}, 15^{\circ}$, and $20^{\circ}$. A previous study investigated the effects of forced foot hyper pronation LE and pelvic alignment. There was a significant increase in internal shank rotation, hip internal rotation and anterior pelvic tilt. An effective correlation between the segmental alignments in every two consecutive types in all levels was observed [3]. An Increase in inclination angle by $10^{\circ}$ is sufficient to cause a change in the shank, left average of $2.33^{\circ}$, and right of $2.44^{\circ}$ has been reported to cause internal shank rotation .

The subjects ambulated in two insole conditions: a control wedge at $0^{\circ}$ and a lateral wedge set at $6^{\circ}[12]$. Then the frontal plane angles of the subtalar joints and knee were measured. It is suggested that these changes occur due to a stable subtalar joint, which leads maintenance of bone stability, and also due to stability of the knee joints reinforced by the surrounding soft tissue [3].

A study by Khamis et al. [13] had found the following results of the measurements with changes in alignment from $10^{\circ}, 15^{\circ}$, and $20^{\circ}$ while standing on a scaffold: standing directly on the floor to encourage bilateral excessive pronation on three wedges at $10^{\circ}, 15^{\circ}$, and $20^{\circ}$ for 20 seconds: If the angle of the ankle joint changes from $0^{\circ}-10^{\circ}$, changes in hip internal rotation is turned to the left by $1.37^{\circ}$, and right by $2.09^{\circ}$, and when the angle changes from $10^{\circ}-15^{\circ}$, it is turned to the left by $0.67^{\circ}$, and to the right by $1.38^{\circ}$, and from $15^{\circ}-20^{\circ}$, it is turned to the left by $0.87^{\circ}$ and to the right by $0.74^{\circ}$.
In a previous study using a fixed wedge, the pelvis was tested for associated correlation with other LE structures. With use of the wedge and heel abducted, the hip abduction angle and pelvic anterior tilt increased [3,9]. Eslami et al. [10] completed a study using a foot wedge to observe the pelvis and trunk angle variability during single-limb stance and found the anterior tilt in the horizontal plane to have a change in $0.052^{\circ}$, however was not significant.

In Pinto et al. [14] study used 3-dimensional motion analysis to study the correlation between the pelvic and calcaneal angle. Unilateral and bilateral use of medially tilted wedges produced a significant increase of calcaneal eversion, on the right and left sides. Bilateral and unilateral increases of the calcaneal eversion caused average pelvic anteversion of $1.57^{\circ}$ and $1.41^{\circ}$, respectively. Unilaterally increased everted position generated an average pelvic lateral tilt of $1.46^{\circ}$.

A limitation of this study is that it is difficult to generalize the findings due to the specific age group used. Another limitation is that the footplate was manually moved to the specific angles and the changes in velocity was not consistent. The right subtalar joint and the left ankle was not measured. A comparison of both right and left subtalar joints was not observed. Also, with a wedge placed unilaterally on only the right side, an analysis of the alignment changes on both sides was not observed.

In further studies, a comparison of right and left subtalar joints need to be researched. Also, there needs to be a specific equipment with automated movement for increased consistency to minimize changes in velocity.

Therefore, the observation of the changes in foot abduction with the use of 3-dimensional motion analysis, angle values of each segment of the LE was predictable. There was no significant differences unilaterally in static and dynamic changes, and further studies are needed to find out the changes in the LE bilaterally for more accurate changes in value.

\section{Conflict of Interest}

The authors declared no potential conflicts of interest with respect to the authorship and/or publication of this article.

\section{References}

1. Hinman RS, Bowles KA, Metcalf BB, Wrigley TV, Bennell KL. 
Lateral wedge insoles for medial knee osteoarthritis: effects on lower limb frontal plane biomechanics. Clin Biomech (Bristol, Avon) 2012;27:27-33.

2. Mann R, Inman VT. Phasic activity of intrinsic muscles of the foot. J Bone Joint Surg Am 1964;46:469-81.

3. Khamis S, Yizhar Z. Effect of feet hyperpronation on pelvic alignment in a standing position. Gait Posture 2007;25:127-34.

4. Sell KE, Verity TM, Worrell TW, Pease BJ, Wigglesworth J. Two measurement techniques for assessing subtalar joint position: a reliability study. J Orthop Sports Phys Ther 1994;19:162-7.

5. Tiberio D. The effect of excessive subtalar joint pronation on patellofemoral mechanics: a theoretical model. J Orthop Sports Phys Ther 1987;9:160-5.

6. Bae SS, Lee SY. The effects of on CTA and Q-angle with the different position of the foot in the standing status. J Korean Phys Ther 2002;14:203-25.

7. Reischl SF, Powers CM, Rao S, Perry J. Relationship between foot pronation and rotation of the tibia and femur during walking. Foot Ankle Int 1999;20:513-20.

8. Hong JA, Kim MH, Jung DH, Lim OB, Yi CH. Effect of medial wedge on muscle activity of lower limb in healthy adults during one leg standing. Phys Ther Korean 2011;18:60-6.

9. Tateuchi H, Wada O, Ichihashi N. Effects of calcaneal eversion on three-dimensional kinematics of the hip, pelvis and thorax in unilateral weight bearing. Hum Mov Sci 2011;30:566-73.

10. Eslami M, Tanaka C, Hinse S, Farahpour N, Allard P. Effect of foot wedge positions on lower-limb joints, pelvis and trunk angle variability during single-limb stance. The Foot 2006;16:208-13.

11. Farokhmanesh K, Shirzadian T, Mahboubi M, Shahri MN. Effect of foot hyperpronation on lumbar lordosis and thoracic kyphosis in standing position using 3-dimensional ultrasound-based motion analysis system. Glob J Health Sci 2014;6:254-60.

12. Kakihana W, Akai M, Nakazawa K, Takashima T, Naito K, Torii S. Effects of laterally wedged insoles on knee and subtalar joint moments. Arch Phys Med Rehabil 2005;86:1465-71.

13. Khamis S, Dar G, Peretz C, Yizhar Z. The relationship between foot and pelvic alignment while standing. J Hum Kinet 2015;46: 85-97.

14. Pinto RZ, Souza TR, Trede RG, Kirkwood RN, Figueiredo EM, Fonseca ST. Bilateral and unilateral increases in calcaneal eversion affect pelvic alignment in standing position. Man Ther 2008;13:513-9. 